\title{
Anfütterung und Wachstum der Larven und Jungfische des Hornhechtes Belone belone
}

\author{
H. RosentHal \\ Biologische Anstalt Helgoland (Zentrale); \\ Hamburg 50, Deutscbland (BRD)
}

\begin{abstract}
Feeding and growth of larvae and fry of the gar fish Belone belone. Artificially fertilized Belone belone $\mathrm{L}$. were reared under controlled conditions in the laboratory to a maximum total body length of approximately $10 \mathrm{~cm}$. Percentage of successful prey catching (Artemia nauplii, Cyclopidae) attained high values already at the time when the larvae started to feed. Larvae and fry readily ingested dried food (tetramin, freeze-dried Daphnia) from the surface of the water. Fry of 16 to $25 \mathrm{~mm}$ total length exhibited intensive cannibalism. The larvae hatched at an average total length of $12.5 \mathrm{~mm}$ and attained a total length of $29 \mathrm{~mm}$ after the first 14 days at $22^{\circ} \mathrm{C}$. Thirty-five days after hatching, average length had increased to $45 \mathrm{~mm}$; after 80 days it averaged $93 \mathrm{~mm}$. At $26^{\circ} \mathrm{C}$, the fishes attained an overall length of $90 \mathrm{~mm}$ after 60 days. Survival was almost $100 \%$ in the 5 day period following hatching. After yolk absorption and first food intake, cannibalism occurred; it resulted in more than $60 \%$ mortality within the next 2 week period. Later causes of mortality were fungal infections, partly associated with injuries resulting from cannibalistic behaviour.
\end{abstract}

\section{EINLEITUNG}

In der Literatur finden sich nur wenige und zudem ältere Arbeiten über den Hornhecht Belone belone L. Frühe Entwidklungsstadien wurden von KupFrer (1868), Ryder (1882, Belone longirostris), Wenckebach (1886), Sobotta (1896), ZiegenHAGEN (1896) und Kopsch (1901) beschrieben. Die Autoren untersuchten vorwiegend Fragestellungen der klassischen Entwicklungsphysiologie, insbesondere die in damaliger Zeit umstrittene Frage der Entstehung der Dottersackentoblasten. Angaben zur Biologie von Belone belone finden sich bei LILLJEBorg (1891) und EHRENBAUM $(1904,1936)$ und Sewerzow (1939, zitiert nach Nikolsky 1957, pp. 364-365). Nahrungsuntersuchungen wurden von SErRlis (1939) an Hornhechten des Mittelmeeres durchgeführt. Hempel (1953) prüfte meristische Merkmale an Exemplaren aus dem Jadebusen und dem Mittelmeer. Verbreitung und Wanderung von Belone belone euxini GüNTHER in den Küstengebieten des Asowschen Meeres konnten von Avedikova (1964) auf Grund von Fanganalysen dargestellt werden. Ober die Vorkommen im Schwarzen Meer berichtete SLASTENENKo (1936).

Der vorliegende Beitrag befaßt sich mit der Aufzucht des Hornhechtes vom Zeitpunkt der ersten Nahrungsaufnahme an bis zum Jungfisch von annähernd $10 \mathrm{~cm}$ Total- 
länge. Über die bei diesen Versuchen gemachten Beobachtungen zur Embryonal- und Larvalentwicklung wird ebenso wie über die Nahrungsausnutzung der Jungfische an anderer Stelle berichtet werden (Rosentral \& Fond, Rosenthal \& PAFFenhöfer, in Vorbereitung).

\section{MATERIAL UND METHODEN}

Die Elterntiere wurden am 27. und 28. Mai 1970 mit dem Stellnetz im Wattenmeer der Insel Texel (Holland) gefangen. Die männlichen Tiere dominierten über wenige laichreife weibliche Fische (vgl. auch HEMPEL 1953). Für die Befruchtungsversuche sind nur fließend reife Tiere benutzt worden. Eier und Sperma wurden unmittelbar nach der Anlandung (etwa 15-20 min nach dem Fang) in Plastikbeutel abgestreift. Die Befruchtung wurde "halbtrocken" vorgenommen, d. h. dem Sperma wurde vor der Verwendung ein etwa gleich großer Volumenanteil Seewasser zugesetzt. Der Befruchtungserfolg lag bei $98 \%$. Nach einer Kontaktdauer von etwa 30 Minuten wurden die Eier durch intensives Spülen mit frischem Seewasser vom anhaftenden Sperma befreit und anschließend auf einem feinmaschigen Kunststoffgitter (Maschenweite etwa $2 \mathrm{~mm}$ ) ausgebreitet. Die Eier verankerten sich mit ihren Haftfäden innerhalb von 1 bis 2 Stunden an diesem Substrat und untereinander zu einem zusammenhängenden Teppich. Klumpenbildung konnte nicht immer vermieden werden.

Etwa 24 Stunden nach der Befruchtung wurde das Eimaterial in $\mathrm{O}_{2}$-überschichteten Thermobehältern nach Hamburg überführt (Transportzeit etwa 6 Std.) und bei

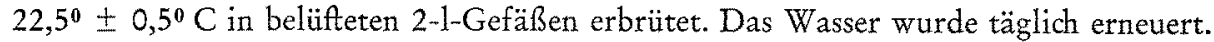
In Anlehnung an Shelbourne (1963) wurden die Eier während der Inkubationszeit mit Antibiotica behandelt ( $25 \mathrm{mg} / 1$ Penicillin; $25 \mathrm{mg} / 1$ Streptomycin-Sulfat). Die Anfütterungsversuche sind in einer fensterlosen Klimakammer bei $22^{\circ} \mathrm{C}$ in schwarzwandigen runden Kunststoffbehältern durchgeführt worden (Abmessungen: $\phi=53 \mathrm{~cm}$, Höhe $=34 \mathrm{~cm}$ ). Angeregt durch die Versuche von PyLE (1969) wurden die Jungfische im Dauerhell (Ringleuchte, Sylvania FCI 6T' 10-WW-RS, 40 Watt) aufgezogen. Ein weiterer Aufzuchtversuch wurde bei $26^{\circ} \mathrm{C}$ im Tageslicht durchgeführt. Die Hälterungsgefäße (Akkugläser, $\phi 35 \mathrm{~cm}$, Höhe $35 \mathrm{~cm}$ ) waren mit einem Bodenfilter nach GREVE (1968) ausgestattet. Etwa $1 / 3$ der Wassermenge wurde täglich erneuert (Salzgehalt 27 bis $30 \%$ ).

In der Klimakammer wurden 9 Aufzuchtbecken über einen geschlossenen Seewasserkreislauf mit aufbereitetem Wasser versorgt (mechanische Filter, Ozonbehandlung, Sedimentationsbecken, Belüftung im Vorratsbecken, Umlauf $1 \mathrm{~m}^{3} / \mathrm{Std}$.). Der Wasserzulauf erfolgte tangential. Auf diese Weise wurde eine schwache Kreisströmung erzielt. Die einzelnen Rundbecken waren zusätzlich mit einem luftgetriebenen Innenflter ausgestattet, so daß beim Auftreten von Krankheiten jedes Aufzuchtbecken zur Verhinderung der Infektionsausbreitung für sich vom Kreislauf abgetrennt werden konnte, ohne dabei die Versorgung der Tiere mit sauerstoffreichem Wasser ernstlich zu gefährden. Von dieser Möglichkeit mußte wiederholt Gebrauch gemacht werden. In den vom Kreislauf abgetrennten Becken wurden täglich 10-12 I Seewasser erneuert und die Kreisströmung über kleine Kreiselpumpen gesteuert. 


\section{ERGEBNISSE}

\section{Verhalten der Larven und Jungfische}

Die Dottersacklarven halten sich nach dem Schlüpfen in der Regel in Oberflächennähe auf. Sie bilden dabei häufig größere Ansammlungen von 50 bis 150 Tieren. Auffällig ist, daß sich die Larven in diesen Aggregationen mit dem Schwanzende zur Mitte hin einstellen (Abb. 1). Sie halten dabei keine Individualabstände ein; Körperkontakte sind die Regel. Es können mehrere solcher Ansammlungen in wenigen Zentimetern Abstand gebildet werden ( $\mathrm{Abb} .1 \mathrm{a}$ ), die sich oft an der Peripherie berühren und dann zu größeren Aggregationen verschmelzen. In Anlehnung an MaGnus (1967) können solche Anhäufungen als "Igelstellungen" bezeichnet werden. Oft wurde beobachtet, daß eine Reihe von Individuen von einer Ansammlung zur anderen hinüberwechselte. Wie aus den Abbildungen $1 \mathrm{a}, \mathrm{b}$ und 2 a ersichtlich, ist der Zusammenhang der Igelstellung weitgehend unabhängig von der Lichtintensität und der Lichteinfallsrichtung. In den schwarzwandigen, von oben beleuchteten Aufzuchtbecken traten sie ebenso häufig auf wie in allseits ausgeleuchteten Glasgefäßen.

Kurz vor Beginn der ersten Nahrungsaufnahme wird der Zusammenhalt innerhalb der Aggregationen schwächer. Die Brut steht vorwiegend im lockeren Verband (Abb. 2b). Gleichzeitig beginnt sie allmählich ihre Schwimmaktivität zu steigern. $\mathrm{Da}$ sie positiv phototaktisch reagiert, wirken die über den Aufzuchtbecken installierten Ringleuchten als "optische Leitlinien“, denen sie folgt (Abb. 2c). Dieses Verhalten täuscht echtes Schwarmverhalten vor. Ein einfacher Versuch verdeutlicht dies: Wird die Ringleuchte ausgeschaltet, so schwimmen die Tiere sofort kreuz und quer durcheinander. Nach dem Einschalten der Lichtquelle stellen sie sich wieder in wenigen Sekunden auf den Rundkurs ein. Sie sind auf diese Weise leicht von den Wänden der Zuchtbecken fernzuhalten.

\section{Anfütterung der Larven}

Der Dottersack wird bei $22^{\circ} \mathrm{C}$ Hälterungstemperatur innerhalb von $36-48$ Stunden nach dem Schlüpfen aufgezehrt. Die Larven schnappen jedoch schon vorher nach Nahrung. Nach den bisherigen Beobachtungen bei der Anfütterung von Fischbrut erreichen die Larven bei den Beutefanghandlungen nur eine sehr geringe Anfangstreffsicherheit, die je nach Fischart etwa zwischen 1 und 10\% liegt (Braum 1963, 1964: Esox lucius, Coregonus; Riley 1966: Pleuronectes platessa; Blaxter 1962, 1966, 1968; Rosenthal 1969a, b: Clupea harengus; Rosenthal 1966: Solea solea; Fonds 1970: Pomatochistus spec.). Hornhechtlarven zeichnen sich demgegenüber zur Zeit der ersten Nahrungsaufnahme durch eine außerordentlich hohe Anfangstreffsicherheit aus. Beim Schnappvorgang können die Larven direkt zubeißen, an großen treibenden Nahrungsbrocken "knabbern", langgestreckte Nahrungsbrocken (Tubifex, Enchytraeus) im Maul wenden und schließlich aus treibenden Kunstfutterflocken Teile abbeißen.

Zur Zeit der ersten Nahrungsaufnahme lag der Schnapperfolg auf ArtemiaNauplien bei $100 \%$, während Süßwassercyclopiden, die in Seewasser wenige Minuten 


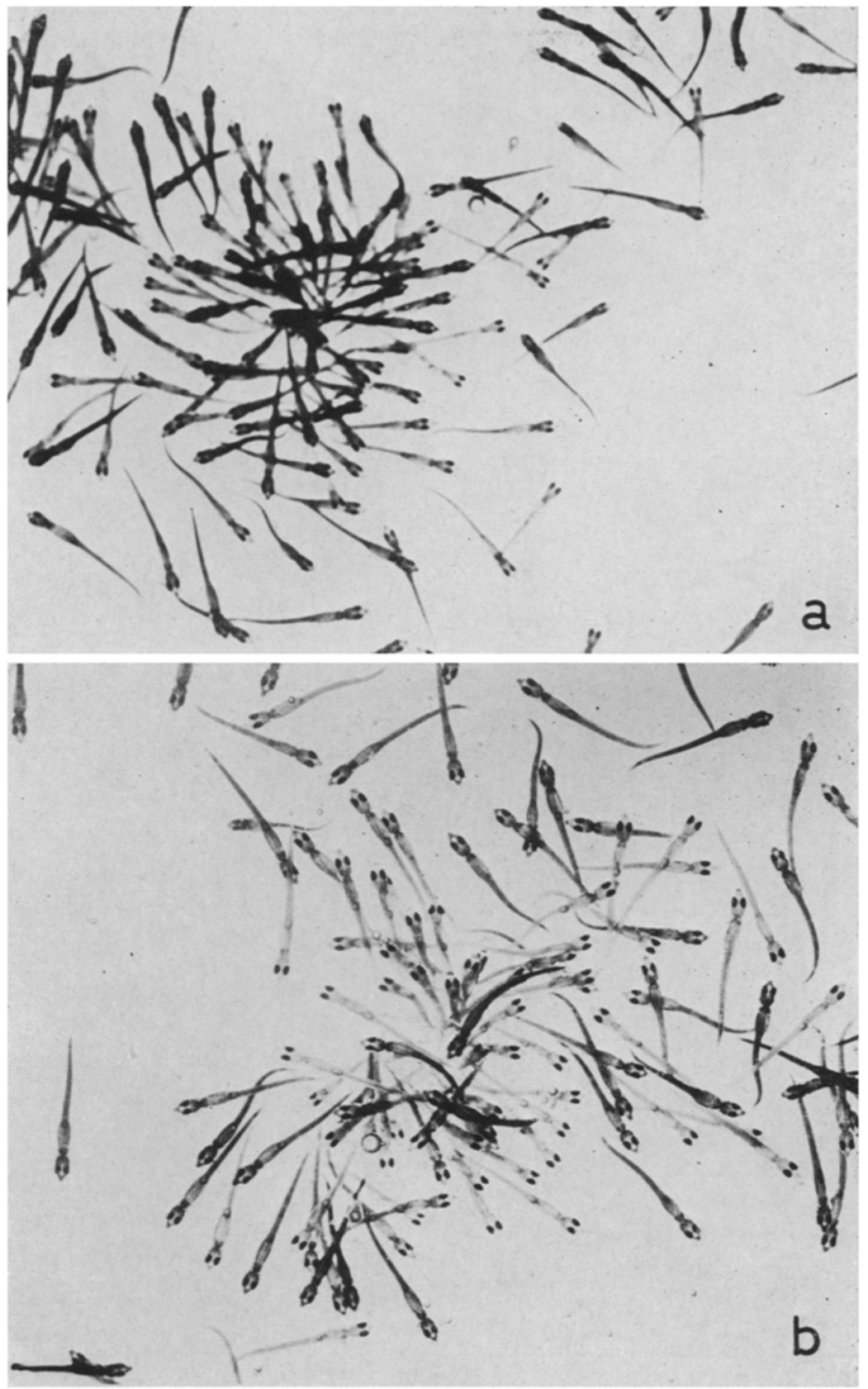

Abb. 1: Belone belone. "Igelstellungen" der Dottersadklarven im Oberflächenwasser eines allseits beleuchteten Glasgefäßes, $a=$ sehr konzentriert stehende Gruppe kurz vor der Verschmelzung mit einer $\mathrm{zweiten}$ (linker Bildrand). $\mathrm{b}=$ aufgelodkerte Igelstellung mit zu- und abwandernden Tieren 

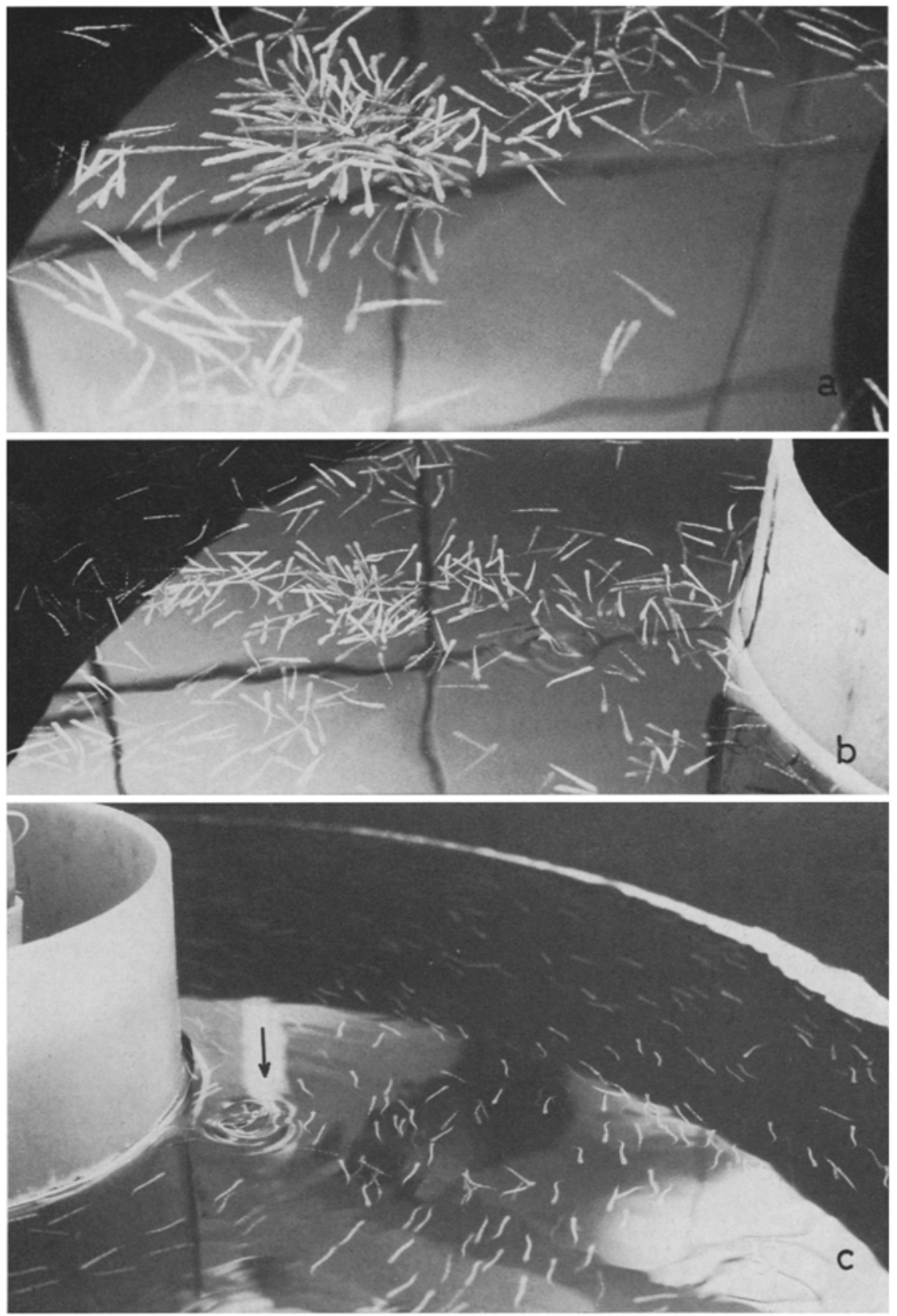

Abb. 2: Belone belone. Verhalten der Larven und Jungfische in schwarzrandigen Arena-Becken. $\mathrm{a}=$ Igelstellung der Dottersacklarven; $\mathrm{b}=$ Auflockerung des Zusammenhalts gegen Ende der Dottersackphase; $c=$ "schwärmende ${ }^{*}$ Jungfische $z$ wischen 15 und $18 \mathrm{~mm}$ Totellänge; Pfeil $=$ springender Fisch; schwarze Linien = Spiegelung der gekachelten Wand der Klimakammer an der Wasseroberfläche 
lebensfähig blieben, mit einer Treffsicherheit von 60 bis $80 \%$ aufgenommen wurden. Schon bei den ersten Beutefanghandlungen sind die Larven des Hornhechtes von keinem bestimmten Beuteschema abhängig. Sie reagieren auf die verschiedensten Partikelformen. So wurde auch Trockenfutter - vorher in einem Mörser zerstoßen - rasch aufgenommen. Während nach Lebendfutter (Cyclopiden, Daphnien, Tubifex, Enchyträen) vorwiegend unterhalb der Wasseroberfläche bis zu einer Tiefe von $10 \mathrm{~cm}$ gejagt wird, nehmen die Jungfische das Kunstfutter (Tetramin, Forellenbrutfutter, gefriergetrocknete Mückenlarven) am günstigsten direkt von der Oberfläche her auf.

\section{Wachstum}

Obwohl die Fütterungsbedingungen - wie bereits erwähnt - nicht optimal waren, konnte ein beachtliches Wachstum erzielt werden. Die Larven schlüpften bei 12,5 $\pm 0,37 \mathrm{~mm}$ Totallänge. Sie besitzen beim Schlüpfen noch kurze Kiefer. Bei einer

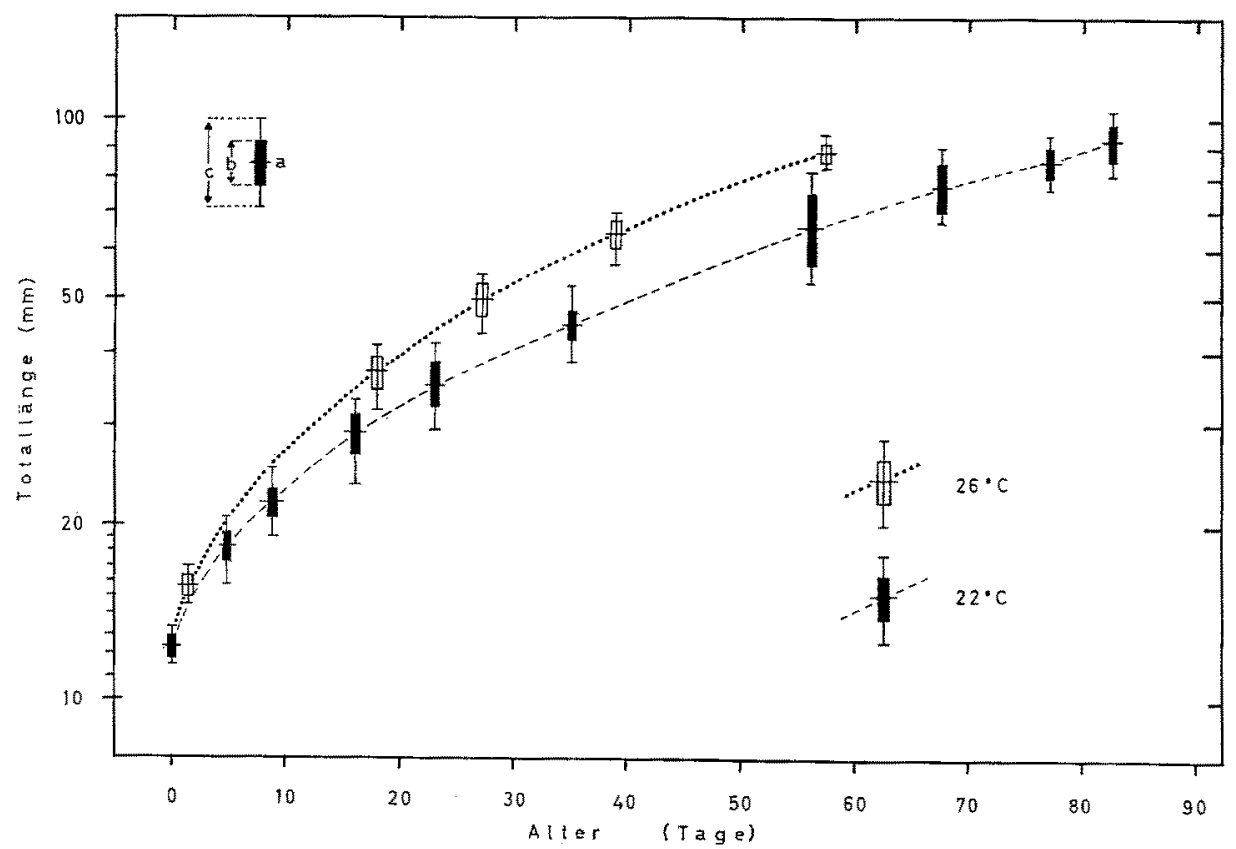

Abb. 3: Belone belone, Längenwachstum (Totallänge) bei $22^{\circ}$ und $26^{\circ} \mathrm{C}$ Aufzuchttemperatur. $a=$ Mittelwert $; b=$ Standardabweichung; $c=$ Variationsbreite

Temperatur von $22^{\circ} \mathrm{C}$ erreichten sie nach 5 Tagen $18,5 \pm 0,9 \mathrm{~mm}$, nach 14 Tagen bereits $29,1 \pm 2,6 \mathrm{~mm}$ und nach 82 Tagen 93,0 $\pm 6,1 \mathrm{~mm}$. Bei einer Aufzuchttemperatur. von $26^{\circ} \mathrm{C}$ wurden $90 \mathrm{~mm}$ Totallänge schon nach 60 Tagen erzielt (Abb. 3). Das rasche Längenwachstum wird besonders durch das Vorwachsen des Unterkiefers betont (Abb. 4), der bis zu $50 \mathrm{~mm}$ über den Oberkiefer hinaus vorragen kann (Hemirhamphus- 


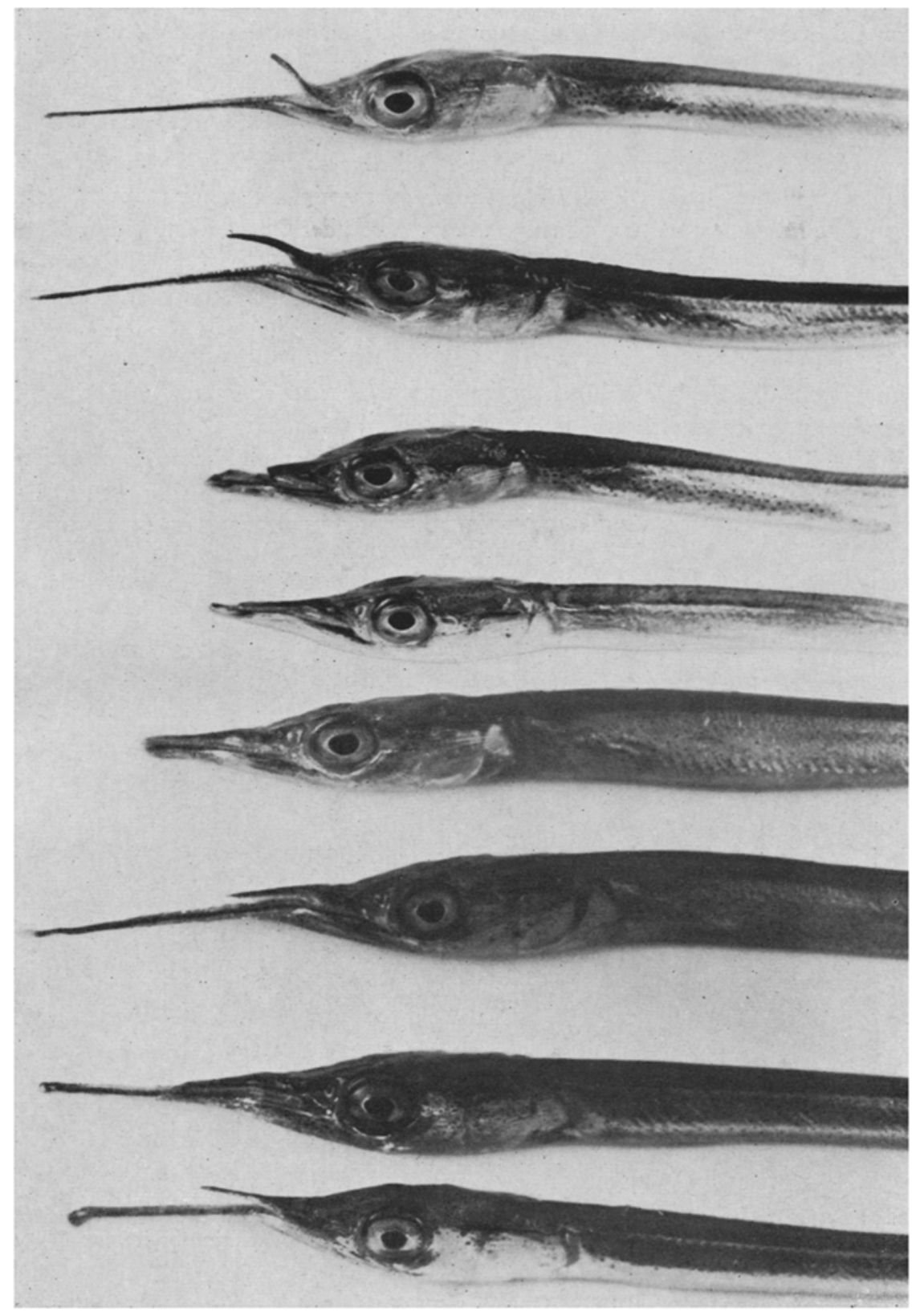

Abb. 4: Belone belone. Jungfische. Deformationen der Kiefer im Aufzuchtversuch; aufgebogene Oberkiefer (oben); abgebrochene und abgewetzte Kiefer (Mitte); leichte Verletzungen am Unterkiefer (unten) 


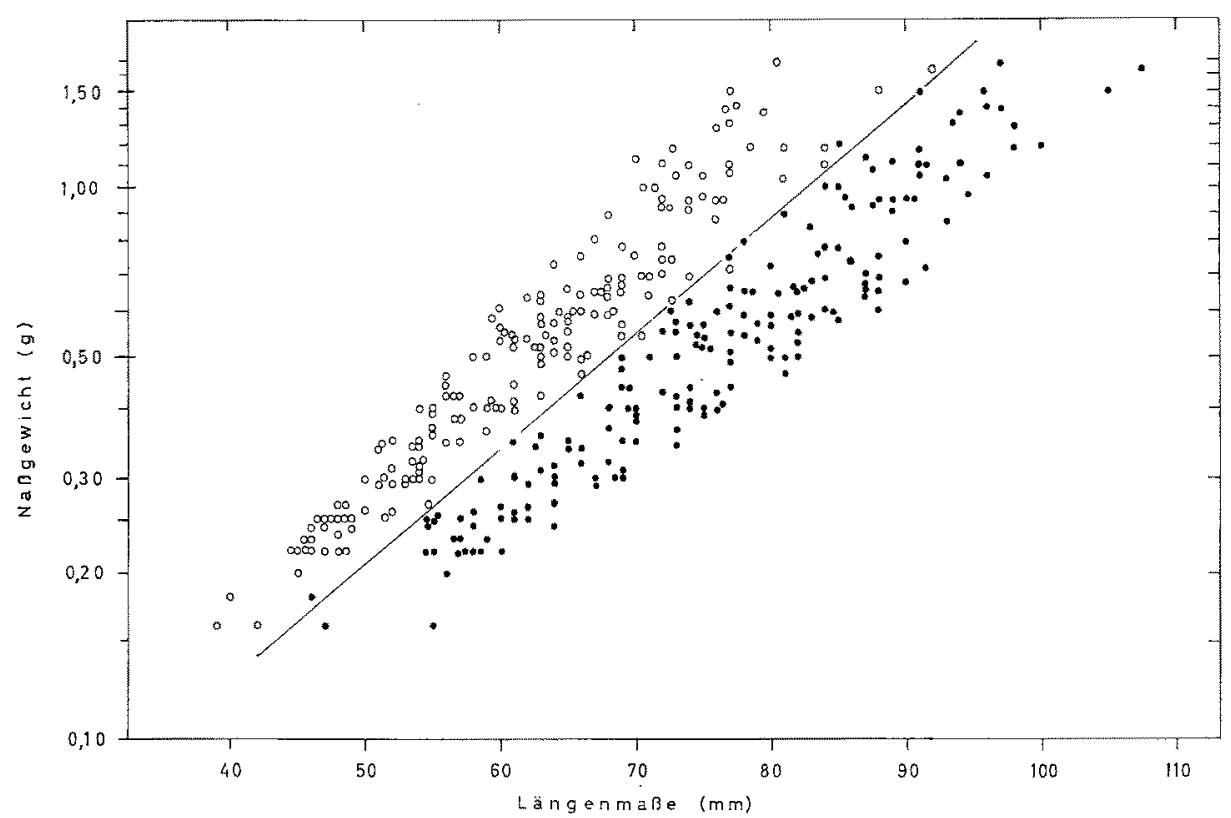

Abb. 5: Belone belone. Längen-Gewichts-Relation, bezogen auf die Totallänge (Punkte) und auf den Abstand zwischen vorderem Augenrand und äußerster Schwanzspitze (Kreise)

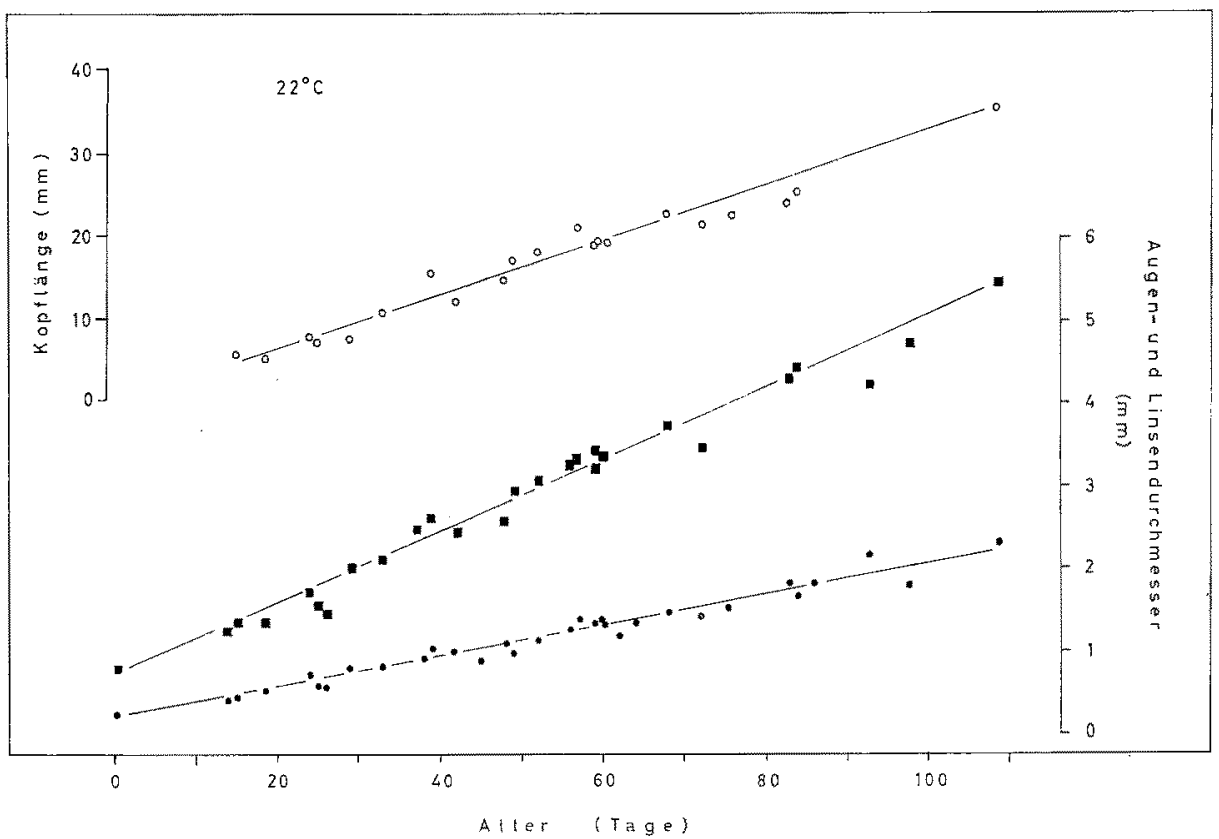

Abb. 6: Belone belone. Kopflänge (Oberkieferspitze bis Operculumrand), Augen- und Linsendurchmesser in Abhängigkeit vom Alter der Jungfische 
Stadium). Die Längengewichtskurve zeigt daher für relativ große Tiere nur geringe Naßgewichte (Abb. 5, Punkte). So wird ein Naßgewicht von 1 bis $1,5 \mathrm{~g}$ meist erst bei Totallängen zwischen 85 und $105 \mathrm{~mm}$ erreicht. Die Werte streuen außerdem erheblich, zumal die Verkrüppelungen des "Schnabels“ die Ergebnisse teilweise verfälschen (Abb. 4). Es wurde daher versucht, neben der Totallänge noch ein anderes Maß zur Darstellung der Längen-Gewichts-Relation zu finden, das diese Unregelmäßigkeit weitgehend eliminiert. Da auch der Oberkiefer oftmals verbogen oder verkürzt war, wurde der vordere Augenrand als Ansatzpunkt für die Längenmessungen gewählt. Das Längen-Gewichts-Verhältnis unter Berücksichtigung der Körperlänge zwischen dem vorderen Augenrand und der äußersten Schwanzspitze ist in Abbildung 5 durch Kreise dargestellt. Augendurchmesser und Kopflänge wachsen im gleichen Verhältnis (Abb. 6), jedoch nicht so schnell wie die Totallänge an. EHrenbaum (1904) erzielte bei Aufzuchtversuchen mit künstlich erbrïteten Larven in 8 Wochen Totallängen bis zu $50 \mathrm{~mm}$.

\section{Sterblichkeit}

Während der Dottersackphase war keine Larvensterblichkeit zu beobachten. Da alle Larven zur ersten Nahrungsaufnahme kamen, gab es auch zu diesem Zeitpunkt

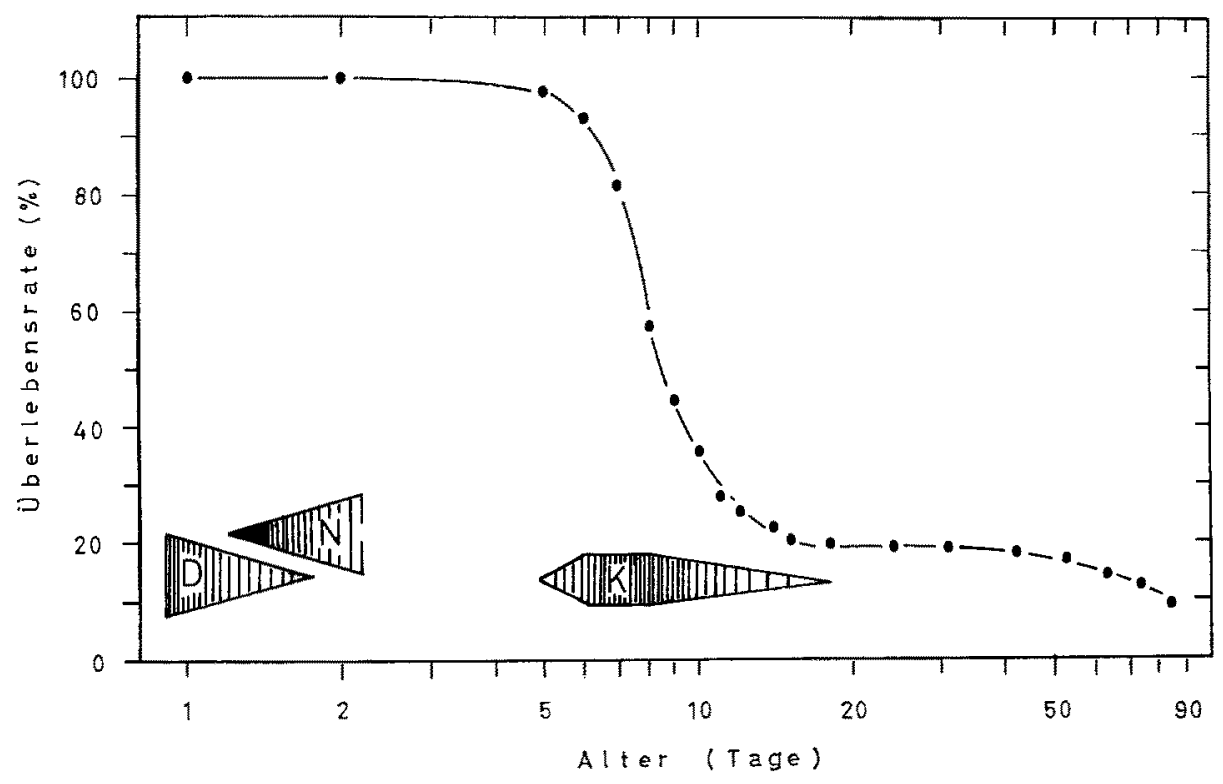

Abb. 7: Belone belone. Oberlebensrate der Larven und Jungfische im Aufzuchtversuch. $\mathrm{D}=$ Dottersackphase, $\mathrm{N}=$ Beginn der Nahrungsaufnahme, $\mathrm{K}=$ Kannibalismus

keine spürbare Sterblichkeit. Eine deutliche Reduzierung der Individuenzahl wurde erst bei Totallängen zwischen 16 und $25 \mathrm{~mm}$ festgestellt. Verantwortlich hierfür war die steigende Aggressivität der Tiere, die in einen ausgeprägten Kannibalismus mün- 
dete. Wenn es einem Jungfisch auch nur selten gelang, einen etwa gleich großen Artgenossen zu verschlingen, so führten doch meist schon die Bißverletzungen (Abb. 8) zum Absterben innerhalb der nächsten 24 bis 48 Stunden.

Nach größeren Fütterungspausen ( 6 bis 8 Std.) traten Verluste dadurch auf, daß zwei Tiere gleichzeitig nach demselben Nahrungsbrocken schnappten und sich mit ihren stark bezahnten Kiefern ineinander verkeilten. Häufig wurden solche Kollisionen trotz starker Kieferdeformationen auch gut überstanden, wie die Beispiele in Abbildung 4

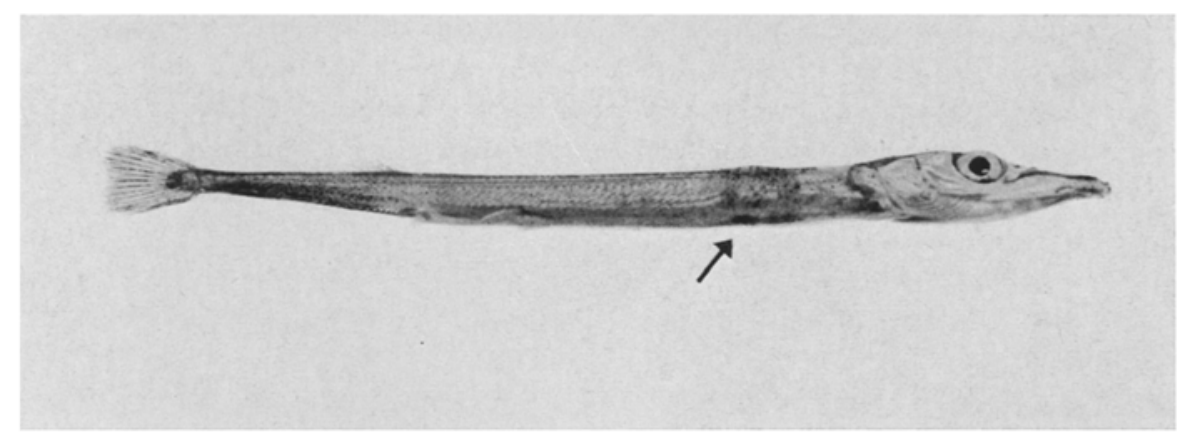

Abb. 8: Belone belone. Bißverletzung (Pfeil) bei einem etwa $70 \mathrm{~mm}$ langen Jungfisch

veranschaulichen. Hohe Sterblichkeiten wurden zeitweise durch Verpilzung der Epidermis sowie durch parasitäre Ciliaten verursacht. Einige Verluste beruhen auf verfahrenstechnischen Fehlern, die bei der Berechnung der Sterblichlseitsraten (Abb. 7) ebensowenig berücksichtigt wurden wie die durch Probeentnahme zu verzeichnenden Verluste. So sprangen gelegentlich Tiere über den Bedkenrand, weil der Wasserstand zu hoch eingestellt war. Andere schlüpften durch beschädigte oder nicht genügend abgedeckte Austrittsöffnungen in den Kreislauf. Die aus personellen Gründen diskontinuierlich durchgeführte Fütterung hatte anscheinend indirekt Einfluß auf die Sterblichkeitsrate. Im leeren Darmkanal wird zumindest bei Jungfischen die peritrophe Membran, in die die Faeces eingeschlossen werden, kontinuierlich weiterproduziert und als fadenförmiger weißer Schlauch fortgesetzt abgegeben. Eine rasche Schwächung der Tiere durch ständigen Substanzverlust macht sie für Infektionen besonders anfällig.

\section{DISKUSSION}

Die vorliegenden orientierenden Versuche haben gezeigt, daß es möglich ist, den Hornhecht nahezu verlustlos iber den kritischen Zeitpunkt der ersten Nahrungsaufnahme hinaus aufzuziehen, wobei die Anfütterung auch mit Kunstfutter vorgenommen werden kann. Die Reduzierung der Sterblichkeit durch Kannibalismus erwies sich als eines der schwierigsten Probleme. $\mathrm{Da}$ der Hornhecht als ein in kleineren Schwärmen gesellig lebender Wanderfisch bekannt ist, wurde zu Beginn der Anzuchtversuche nicht mit einer kannibalischen Phase gerechnet. Maßnahmen dagegen konnten nur improvisiert werden. In einigen Aufzuchtbecken gelang es, den Kannibalismus durch unter- 
schiedlich starke Kreisströmungen spürbar einzudämmen. Eine optimale Strömungsgeschwindigkeit kann aus diesen geringen Beobachtungswerten nicht abgelesen werden. Es besteht die Hoffnung, daß anlagentechnische Verbesserungen diese Art der Sterblichkeit wesentlich reduzieren helfen. Spätere Verluste durch Bißverletzungen wurden fast ausschließlich von Vorwüchsern verursacht. Es wird zu prüfen sein, ob das Wachstum von einer Größenhierarchie beeinflußt wird (NAGoshI 1967). Ein Raumfaktor (vgl. Wirler \& SChNIGENBerg 1927) scheint für die Wachstumsgeschwindigkeit des Hornhechts ohne Bedeutung zu sein (SENGBusCH et al. 1967). Die bei $26^{\circ} \mathrm{C}$ aufgezogenen Tiere wuchsen in wesentlich kleineren Behältern und bei etwa gleichem Nahrungsangebot schneller als in den größeren, bei $22^{\circ} \mathrm{C}$ temperierten Zuchtbecken. Die von EHRENBAUM (1904) gefundenen Wachstumsdaten sind wesentlich niedriger als die im vorliegenden Aufzuchtversuch erzielten Werte. Leider ist die von EHRENBAUM benutzte Anzuchttemperatur nicht bekannt und ein Vergleich der Ergebnisse daher nicht möglich.

Eine klare Deutung des eigenartigen Verhaltens der Dottersacklarven, sich zu "Igelstellungen" zusammenzuziehen, kann nicht gegeben werden. Die Annahme einer Seeigelmimikry erscheint wenig sinnvoll. Magnus (1967) beschreibt ähnliche Verhaltensweisen bei einem Jungfisch-Schwarm des Korallenwelses Plotosus anguillaris. Nach seinen Beobachtungen wird die „Igelstellung“ nur bei Bedrohung unter unnormalen Verhältnissen, wie dem Gefangensein in einem Ebbetümpel, eingenommen. Eine Deutung dieses Verhaltens als Fluchtintensionsbewegung wäre auch beim Hornhecht denkbar.

Als außerordentlich günstig für die Aufzudhtchancen junger Hornhechte muß die Tatsache bezeichnet werden, daß die Verkrüppelungen des vorwachsenden Unterkiefers zu keinen Infektionen geführt haben und die Tiere auch kaum bei der Nahrungsaufnahme behindert waren.

Um eine optimale Nutzung des Kunstfutters zu erzielen, wäre die Entwicklung eines geeigneten Schwimmfutters erforderlich, da die Jungfische des Hornhechtes dieses besonders gern von der Oberfläche her aufnehmen, absinkenden Nahrungsbrocken jedoch nicht nachschwimmen. Die ausschließliche Verwendung von Kunstfutter würde auch den Einsatz eines Futterautomaten ermöglichen und das Nahrungsangebot optimieren helfen.

\section{ZUSAMMENFASSUNG}

1. Belone belone L. wurde von der künstlichen Befruchtung an bis zu einer Totallänge von annähernd $10 \mathrm{~cm}$ unter kontrollierten Laborbedingungen aufgezogen.

2. Dottersacklarven zeigten unter verschiedenen Lichtbedingungen "Igelstellungen", die in Anlehnung an Magnus (1967) als Fluchtintensionsbewegung interpretiert werden.

3. Die Beutefanghandlungen erreichen schon zur Zeit der ersten Nahrungsaufnahme eine außerordentlich hohe Anfangstreff sicherheit.

4. Larven und Jungfische nehmen Kunstfutter sehr gut von der Oberfläche her auf.

5. Bei Totallängen $\mathrm{zwischen} 16$ und $25 \mathrm{~mm}$ neigen die Jungfische besonders stark zum Kannibalismus. 
6. Die mit $12,5 \mathrm{~mm}$ Totallänge schlüpfenden Larven wuchsen bei $22^{\circ} \mathrm{C}$ in den ersten 14 Tagen im Mittel bis auf $29 \mathrm{~mm}$, innerhalb von 35 Tagen auf $45 \mathrm{~mm}$ und in 80 Tagen auf etwa $93 \mathrm{~mm}$ heran. Bei einer Aufzuchttemperatur von $26^{\circ} \mathrm{C}$ wurden $90 \mathrm{~mm}$ Totallänge bereits nach 60 Tagen erreicht.

7. Die Überlebensrate betrug während der Dottersackphase und zur Zeit der ersten Nahrungsaufnahme annähernd $100 \%$. Die durch Kannibalismus verursachte Sterblichkeit erreichte mehr als $60 \%$. Erhebliche Verluste wurden dabei sekundär durch Pilzinfektionen und parasitäre Ciliaten verursacht.

Danksagungen. Herrn Dr. M. Fonds (Nederlands Instituut voor Onderzoek der Zee, Texel) sowie den Herren $H$. Beumkes und M. BinsBergen (Rijksinstituut voor Visserijonderzoek, Texel) danke ich für die freundliche Unterstützung bei der Materialbeschaffung. Ferner gilt mein Dank den Herren Dr. G.-A. Pafrenhröfer, Dipl.-Ing. G. Luther, G. Lauckner und H. KuHLMANN sowie meiner Assistentin Frau G. Fürstenderg für vielseitige Hilfen während der Durchführung der Versuche.

\section{ZITIERTE LITERATUR}

Avedikova, T.M., 1964. On the distribution and migrations of Belone belone emxini GüNTHer. Vop. Ikhtiol. 4, 753-756.

BLAXTER, J. H. S., 1962. Herring rearing IV. Rearing beyond the yolk sac stage. Mar. Res. 1, $1-18$.

- 1966. The effect of light intensity on the feeding ecology of herring. In: Light as an ecological factor. Ed. by R. Bainbridge, G. C. Evans \& D. Rackham. Blackwell, Oxford. Symp. Br. ecol. Soc. 6, 393-409.

- 1968. Rearing herring larvae to metamorphosis and beyond. J. mar. biol. A.ss. U.K. 48, $17-28$.

BraUM, E., 1962. Erste Nahrungsaufnahme bei Blaufelchen (Coregonus wartmanni BLOCH) und Hecht-Jungfischen (Esox lucius L.) des Bodensees. Naturwissenschaften 49, 189.

- 1964. Experimentelle Untersuchungen zur ersten Nahrungsaufnahme und Biologie an Jungfischen von Blaufelchen (Coregonus wartmanni Broch), Weißfelchen (Coregonus fera JuRINE) und Hechten (Esox lucius L.). Arch. Hydrobiol. (Suppl. Bd) 28, 183-244.

Ehrenbaum, E., 1904. Eier und Larven von Fischen der Deutschen Bucht. III. Fische mit festsitzenden Eiern. Wiss. Meeresunters. (Abt. Helgoland) 6, 127-200.

- 1936. Naturgeschichte und wirtschafliche Bedeutung der Seefische Nordeuropas. Schweizerbart, Stuttgart. 337 pp.

Fonds, M., 1970. Remarks on the rearing of gobies (Pomatoschistus minutus and P. lozanoi) for experimental purposes. Helgoländer wiss. Meeresunters. 20, 620-628.

Greve, W., 1968. The "planktonkreisel", a new device for culturing zooplankton. Mar. Biol. 1, 201-203.

Hempei, G., 1953. Meristische Merkmale von Hornhechten (Belone acus Rrsso) aus dem Jadebusen und dem Golf von Neapel. Kurze Mitt. fischbiol. Abt. Max-Planck-Inst. Meeresbiol. Wilhelmsh. 2, 20-23.

Korsch, F., 1901. Die Entstehung des Dottersackentoblasts und die Furchung bei Belone acus. Int. Mschr. Anat. Physiol, 18, 43.

KupfFer, C., 1868. Beobachtungen über die Entwicklung der Knochenfische. Arch. mikroskop. Anat. EntwMech. 4, 209-272.

LiLljeboRG, W., 1891. Sxeriges och Norges Fauna. Schulz, Uppsala, 2, 1-788.

MaGNus, D. B. E., 1968. Zur Deutung der Igelstellung beim Jungfisch-Schwarm des Korallenwelses, Plotosus anguillares (Bloch) (Pisces, Nematognathi, Plotosidae), im Biotop. Zool. Anz. (Suppl. Bd) 31, 402-409. 
Nagoshi, M., 1967. Experiments on the effects of size hierarchy upon the growth of guppy (Lebistes reticulatus). J. Fac. Fish. pref. Univ. Mie-Tsu 7 (2), 165-189.

Nikolsky, G. W., 1957. Spezielle Fischkunde. Dt. Verl. Wiss., Berlin. 632 pp.

PyLE, E. A., 1969. The effect of constant light or constant darkness on the growth and sexual maturity of brook trout. Cortland Hatchery Report 36 for the year 1967. Fish. Res. Bull. N.Y. 31, 13-19.

RuLEY, J. D., 1966. Marine fish culture in Britain, VII. Plaice (Plewronectes platessa L.). Postlarval feeding on Artemia salina L. nauplii and the effects of varying feeding levels. J. Cons. perm. int. Explor. Mer 30, 204-221.

Rosenthal, H., 1966. Beobachtungen ïber das Verhalten der Seezungenbrut. Helgoländer wiss. Meeresunters. 13, 213-228.

- 1969. Verdauungsgeschwindigkeit, Nahrungswahl und Nahrungsbedarf bei den Larven des Herings, Clupea barengus L. Ber. dt. wiss. Kommn. Meeresforsch. 20, 60-69.

- 1969. Untersuchungen über das Beutefangverhalten bei Larven des Herings Clupea harengus. Mar. Biol. 3, 208-221.

RYDER, J. A., 1882. Development of the Silver gar (Belone longirostris), with observations on the genesis of the blood in embryo fishes, and a comparison of fish ova with those of other vertebrates. Bull. US Fish. Commn 1, 283-301.

SEIRLIS, D. G., 1939. Contributo allo studio dell'alimentazione dei pesci Belone belone LiN. Boll. Pesca Piscic. Idrobiol. 15 (6), 715-726.

Sengbusch, R. von, Mesxe, Ch., Szablewsky, W. \& Lührs, B., 1967. Gewichtszunahme von Karpfen in Kleinstbehältern, zugleich ein Beitrag zur Aufklärung des Raumfaktors. Z. Fisch. 15, 45-60.

Shelbourne, J. E., 1963. A marine fish-rearing experiment using antibiotics. Nature, Lond. $198,74-75$.

- 1964. The artifical propagation of marine fish. Adv. mar. Biol. 2, 1-83.

Siastenenko, E. P., 1936. Notes sur quelques poissons de la mer noire. Annls Scient. Univ. Jassy 22, 297-305.

SовоттA, J., 1896. Zur Entwicklung von Belone acus. Verh. Anat. Ges. Berlin 1896, 93-96.

WENCKEBACH, K. F., 1886. Beiträge zur Entwicklungsgeschichte der Knochenfische. Arch. mikrosk. Anat. EntwMech. 28, 225-251.

Willer, A. \& Schnigenberg, E., 1927. Untersuchungen über das Wachstum bei Fischen. 1. Uber den Einfluß des Raumfaktors auf das Wachstum der Bachforellenbrut. Z. Fisch. 25, 263-290.

ZIEGENHAGEN, H., 1896. Ober Entwidklung der Circulation bei Teleostiern, insbesondere bei Belone. Verh. Anat. Ges. Berlin 1896, 100-108.

Anschrift des Autors:

Dr. H. ROSENTHAL

Biologische Anstalt Helgoland

(Zentrale)

2 Hamburg 50

Palmaille 9

Deutschland (BRD) 